\title{
Ewing's Sarcoma: A Case Report
}

\section{Sarcoma de Ewing. A Propósito de un Caso}

XVIII International Seminar on Health, Food and Human Nutrition

Corresponding Author:

Diana Belén Cuenca Mora

pauly_mariuxi@hotmail.com

Published: 9 September 2021

Production and Hosting by

Knowledge E

(ㄷ) Diana Belén Cuenca Mora et al. This article is distributed under the terms of the Creative Commons Attribution License, which permits unrestricted use and redistribution provided that the original author and source are credited.

\author{
Diana Belén Cuenca Mora1, Tatiana Karolina Miranda Arellano², María \\ Romyna Delli Villavicencio ${ }^{1}$, Erick Patricio Orozco Velasco ${ }^{1}$, and Vicente \\ Xavier Muñoz Salinas ${ }^{1}$ \\ ${ }^{1}$ Médico/a Residente de la Unidad Oncológica SOLCA Esmeraldas y Duchicela, Riobamba, \\ Chimborazo, Ecuador \\ ${ }^{2}$ Médico Rural del Centro de Salud Yaruquíes, Riobamba, Chimborazo, Ecuador
}

\section{Abstract}

Ewing's sarcoma is a bone tumor, considered one of the most aggressive, for its great capacity of metastasizing. Higher incidence in men, appears almost exclusively in the first three decades of life. Affects the long bones in the metaphyseal region, at its proximal end; the most affected are the femur, iliac, tibia, humerus, fibula and ribs; however, there are atypical locations such as in the hands, feet and extraosseous. The clinical case of a 13-year-old male adolescent patient, who two months before his medical attention presented a mass at the level of the second and third right costal arches that increased vertiginously. After routine and laboratory examinations performed at a third level complexity hospital, he was diagnosed with Ewing's Sarcoma, where 3 chemotherapy sessions were performed; however, despite the treatment, he presented brain and bone metastases. Ewing's tumor was highly aggressive and has a bleak prognosis.

Keywords: sarcoma, ewing, tomography, morbid metastasis.

\section{Resumen}

El Sarcoma de Ewing es un tumor óseo, considerado uno de los más agresivos, por su gran capacidad de metástasis. Tiene mayor incidencia en hombres, se presentan de forma casi exclusiva en las tres primeras décadas de vida, afecta a los huesos largos en la región metafisodiafisaria, en su extremo proximal, los más afectados son el fémur, el iliaco, la tibia, el humero, el peroné y las costillas. Sin embargo, existen localizaciones atípicas como en los pies manos y extraóseas. Se presentación el caso de un paciente adolescente masculino de 13 años, que dos meses antes de la atención médica presenta una masa a nivel de segundo y tercer arco costal derecho que aumenta de tamaño de forma vertiginosa. Posterior a exámenes de gabinete y laboratorio realizados en Hospital de Tercer Nivel de Complejidad, es diagnosticado de Sarcoma de Ewing en donde se realizan 3 sesiones de quimioterapia, sin embargo, pese el tratamiento presenta metástasis cerebrales y óseas, El tumor de Ewing es altamente agresivo y tiene un pronóstico sombrío

Palabras Clave: sarcoma de ewing, tomografía, metástasis mórbida 


\section{Introducción}

El Sarcoma de Ewing debe su nombre a su descubridor James Ewing, quien lo estudio en 1918, pero fue hasta 1921 que se conoce su origen histológico. Se presenta aproximadamente en 3 casos por cada millón de habitantes por año. Es un tumor altamente agresivo de origen óseo y de localización en los huesos largos, que se caracteriza por ser una masa heterogénea que por el tejido de origen es sarcoma, que es un tumor de células pequeñas redondas y azules [1].

El manejo es multidisciplinario, los métodos de imagen son claves en el diagnóstico, el tratamiento es básicamente la quimioterapia sistémica neoadyuvante cuyo objetivo es disminuir el tamaño del tumor y la carga tumoral, y en lo posterior se establecen cirugías que preservan las piezas óseas afectadas y la erradicación de la micro metástasis [2].

El sarcoma de Ewing del hueso es el segundo tipo más común de cáncer de hueso primario, superado sólo por el osteosarcoma. En ese momento de diagnóstico, el 24\% de los pacientes tienen enfermedad metastásica, que afecta más comúnmente a los pulmones, seguido de otros huesos y médula ósea [3-5]. Los tumores de la familia del sarcoma de Ewing incluyen el sarcoma de Ewing, los tumores neuroectodérmicos primitivos periféricos y los tumores de Askin. Estos tumores son pequeños tumores de células redondas azules indiferenciados que aparecen principalmente en los huesos y con menos frecuencia en los tejidos [6]. El sarcoma de Ewing es un tumor óseo maligno (que se presenta predominantemente en la pelvis, el fémur, la tibia y las costillas) o un tumor de tejido blando (que se presenta predominantemente en la pared torácica, el músculo glúteo, las cavidades pleurales y los músculos cervicales) que afecta principalmente a los niños, los adolescentes y los adultos jóvenes (AYA), con $\sim 1,5$ casos por millón de niños y AYA a nivel mundial [7-9]. El sarcoma de Ewing en el aspecto pediátrico se caracteriza por la expresión de fusiones quiméricas de factores de transcripción de la familia de EWS y ETS, lo que representa un paradigma para el estudio de los cánceres impulsados por los reordenamientos de los factores de transcripción [8].

La tasa de sobrevida depende la de respuesta de la quimioterapia cuando esta es positiva la supervivencia será de alrededor de 5 años que es casi el doble de las que no respondieron [10]. La recurrencia del sarcoma es consecuencia de la respuesta negativa. Cuando existen metástasis estas serán óseas y pulmonares [11]. Se describe el caso clínico de un paciente a través de su historia clínica para conocer el tratamiento y pronóstico de esta rara patología.

\section{Caso}

\subsection{Información del paciente}

Paciente masculino de 13 años, mestizo, estudiante, católico, producto de segunda gesta, madre se realiza 8 controles prenatales, nace en hospital privado con llanto fuerte e inmediato, sin antecedentes patológicos personales y familiares de importancia. 


\subsection{Hallazgos clínicos}

\subsubsection{Exámen físico general}

Paciente en decúbito dorsal activo, despierto orientado colaborador no refiere molestias con actividad psicomotriz conservada, peso $43 \mathrm{~kg}$, talla $161 \mathrm{~cm}$, temperatura 37 grados centígrados, frecuencia cardiaca 78 , frecuencia respiratoria 20/min, tensión arterial 95/65 mm. Hg., saturación 92 aire ambiente.

\subsubsection{Exámen físico regional}

\section{(i) Cabeza}

Normo cefálico, cabello negro de implantación normal.

Ojos: Pupilas isocóricas normo reactivas a la luz, escleras ligeramente pálidas.

Oídos: Pabellón auricular de implantación normal conducto auditivo externo permeable.

Nariz: Pirámide nasal normal, fosas nasales permeables.

Boca Mucosas orales húmedas, piezas dentales en regular estado general.

\section{(ii) Cuello}

Simétricos movilidad activa y pasiva conservada, no se palpan adenomegalias.

\section{(iii) Tórax}

Asimétrica expansibilidad conservada, presencia de masa de cuarta a séptima costilla aproximadamente $10 \mathrm{~cm}$ de diámetro mayor, la misma que es dura, inmóvil, no dolorosa.

Aparato Respiratorio Pulmones murmullo vesicular disminuido en ápice pulmonar derecho, abolido en el tercio medio y en la base ipsilateral.

Aparato Cardiovascular. Ruidos cardíacos rítmicos no soplos no ruidos sobreañadidos.

\section{(iv) Abdomen}

Suave depresible no doloroso a la palpación no visceromegalias.

\section{(v) Extremidades y sitios de puntura}

Simétricas, fuerza conservada llenado capilar menor a dos segundos. Presencia de adenopatías inguinales bilaterales de 0,5 a $1 \mathrm{~cm}$ no dolorosas. 


\section{(vi) Región inguino genital}

Genitales externos masculinos sin patología.

\subsection{Time line/línea cronológica}

Paciente de 13 años es referido a un hospital Pediatrico desde un Hospital General, madre del paciente indica que su hijo hace aproximadamente, dos años sufre golpe con objeto contusivo contra una reglilla metálica sobre costilla derecha sin complicación aparente pero hace dos meses, observa la presencia de una masa a nivel de tercer y cuarta costilla pequeña no dolorosa, que aumenta de tamaño paulatinamente, produciendo dolor con la movilización, acude al médico quién solicita ecografía de tórax evidenciando masa de seiscentímetros. De largo por 3 de ancho, daño en patrón muscular con calcificaciones por lo que refieren a Hospital de Solca.

16/09/2019. En Hospital de Solca se realiza. Alfafetoproteína y antigeno 4,8 ng/ml. Valor de referencia: $0-10,9 \mathrm{ng} / \mathrm{ml}$.

17/09/2019. Se deriva a hospital de tercer nivel para su manejo integral, con resultados de alfafetoproteína y antígeno, carcinoembrionarios negativos (CEA).

17/10/2019. Se cumple con el ingreso en un hospital de tercer nivel de complejidad. Se realiza tomografía de tórax que reporta derrame pleural en hemitórax derecho con una densidad de $10 \mathrm{UH}$, en su interior se aprecia imagen redondeada de contornos regulares que envuelve séptimo arco costal, la cual ocasiona erosión decidiendo biopsia de masa más toracoscopia.

18/10/2019. Se realiza toracoscopia con hallazgos de masa toráxica derecha de aproximadamente $10 \mathrm{~cm}$ por $13 \mathrm{~cm}$, derrame pleural derecho de aproximadamente $2000 \mathrm{~mL}$, implantes en lóbulo medio. Se coloca tubo toráxico con posterior drenaje de líquido serohemático.

19/10/2019. En radiografía de control persistencia de derrame pleural, aunque con disminución de drenaje se decide mantener el mismo.

16/09/2019. Se realiza prueba CEA.

18/10/2019. Se realiza microbiología muestra líquido pleural.

22/10/2019. Se realiza estudio histopatológico.

\subsection{Evaluación diagnóstica}

\subsubsection{Exámenes de laboratorio}

Microbiología muestra líquido pleural. Resultado citológico descripción macroscópica (se recibe $50 \mathrm{cc}$ de líquido turbioamarillo rojizo, se procesa todo el material en dos placas (Giemsa y Papanicolau) y bloque celular (Hematoxilina \& Eosina). Descripción microscópica frotis y bloque celular constituidas por células pequeñas a medianas con núcleo hipercromático irregular y escaso citoplasma en fondo hemorrágico. Y se emite un diagnóstico. Citología de líquido tumoral: Positivo para malignidad. 
Resultado histopatológico, Descripción macroscópica. (Se reciben tres fragmentos irregulares de tejido blando, que miden entre 0,3 y $1,5 \mathrm{~cm}$ blanquecidos. Se procesa todo el material en un cassette). Descripción microscópica. Los cortes histológicos muestran proliferación difusa y discohesiva de células pequeñas redondas y azules con citoplasma eosinófilo y núcleo redondeado a oval de bordes irregulares y nucleolos inconspicuos focalmente las células se distribuyen alrededor de vasos sanguíneos, se cuentan hasta 21 mitosis en 20 campos de gran aumento.

\subsubsection{Exámen de imagen}

Radiografía Antero Posterior y lateral de tórax ( Rx AP y L ) En campo pulmonar izquierdo se evidencia incremento de la trama vascular. Silueta cardíaca no valorable, ángulos cardio y costo frénicos izquierdo libres. En campo pulmonar derecho se observa presencia de radiopacidad que ocupa gran parte del lóbulo medio y el lóbulo inferior, con de borramiento de los ángulos cardio y costo frénicos ipsilaterales con presencia de imagen radiolúcida en probable relación a hidroneumotóraxa nivel de partes blandas de hemitórax derecho se evidencia presencia de imagen radiopaca de bordes mal definidos. Costillas de parrilla costal derecha impresionan lesiones osteolíticas. Se sugiere correlacionar con hallazgos en TAC (Figura 1).
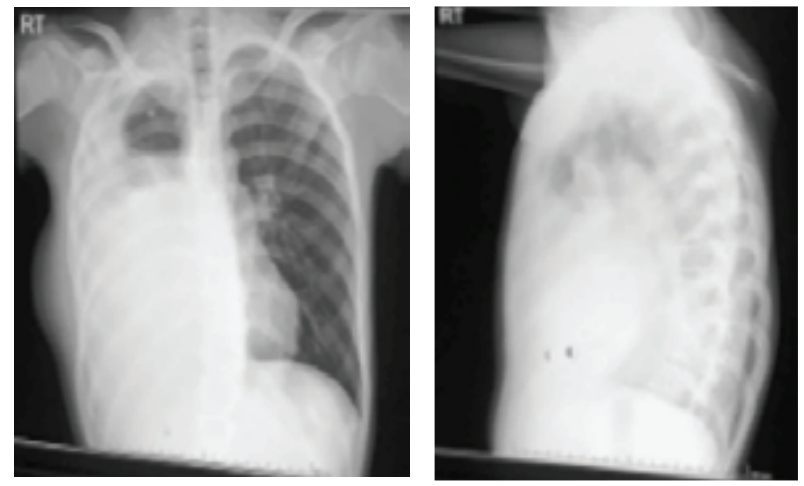

\section{Figure 1}

$R x A P$ de tórax radiopacidad que ocupa gran parte del lóbulo medio y el lóbulo inferior.

Ecografia de Tórax. Se realiza valoración de hemitórax derecho en las regiones anterior, lateral y posterior, evidenciando. A nivel de partes blandas se observa masa sólida de contornos regulares heterogénea con predominio hiperecogénico con escasa vascularidad central que mide $10,7 \times 9,7 \times 11,2 \mathrm{~cm}$ con un volumen aproximado de 618,1 $\mathrm{mL}$ y en el espacio pleural derecho se observa presencia de derrame pleural con finos ecos internos, que mide $12,9 \times 7,7 \times 13,6 \mathrm{~cm}$ con un volumen aproximado de $713,7 \mathrm{~cm}$. TAC simple de cráneo (Figura 2).

Biopsia por toracoscopia en masa de tórax. Se concluye Tumor de células pequeñas, redondas y azules. Hallazgos histopatológicos compatibles con Sarcoma de Ewing. Resultado de histopatología que indica tumor de células pequeñas redondas y azules. Compatibles con Sarcoma de Ewing, decidiendo traslado al servicio de Quimioterapia. 


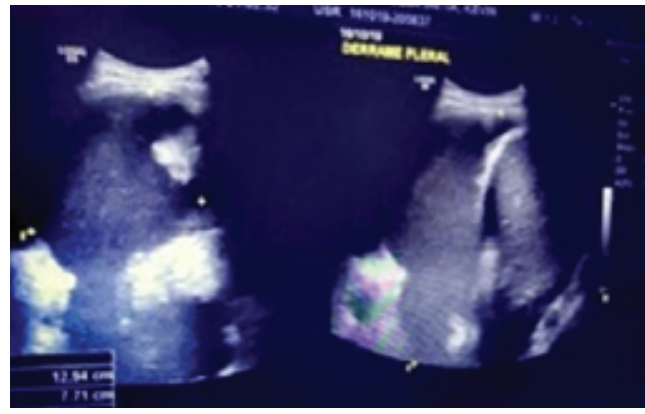

\section{Figure 2}

Ecografía de tórax, se observa una masa sólida de contornos regulares heterogénea.

El diagnóstico clínico esta dado por los exámenes de gabinete y de laboratorio, por lo que se concluye que el paciente tiene Sarcoma de Ewing.

\subsubsection{Diagnóstico diferencial}

Se realizó diagnóstico diferencial por la localización de los tejidos. En las partes musculoesqueléticas se presentan tumores benignos como el rabdomioma maligno rabdomiosarcoma de diferenciación incierta, benigno como los miomas, maligno como histocitoma fibroso angiomatoide, Sarcoma sinovial, sarcoma epiteloide, sarcoma alveolar de partes blandas, sarcoma de partes blandas, Sarcoma de Ewing [12].

\subsubsection{Por la edad del paciente}

Infancia se presentan los rabdomiosarcoma, algunas fibromatosis, Sarcoma de Ewing, Hamartorma mesenquial, entre 15 a 30 años. Sarcomas sinoviales, fibrosarcomas. De 30 años y más sarcoma de partes blandas más frecuente menores de 50 años Liposarcoma. Histocitoma maligno, Fibrosarcoma de partes blandas presenta mejor pronóstico cuando ocurre en la infancia que en la edad adulta [12].

\subsubsection{Pueden presentar tumores posteriores a antecedentes traumáti- $\cos$}

Observarse osificaciones heterotópicas post-traumatismos.Hematomas o pseudoaneurismas después traumatismos penetrantes. Granulomas o infecciones de partes blandas por cuerpos extraños [12].

\subsubsection{Tiempo de evolución y desarrollo de la masa tumoral}

Los tumores indoloros y profundos experimentan mayor crecimiento (liposarcoma o lipomas profundos). Que los tumores superficiales o periféricos (rabdomiosarcoma en niños o neurinoma). El rabdomiosarcoma y el tumor neuroectodérmico primitivo en niños y adultos jóvenes crecen más rápido. El sarcoma sinovial o el sarcoma epitelioide 
se puede presentar de forma insidiosa. Un tumo de tamaño pequeño durante mucho tiempo [13].

\subsubsection{Según el sitio de localización}

Sarcomas de partes blandas: Más localizan de forma frecuente en extremidades inferiores, especialmente en muslos y zona glútea. Sarcoma epitelioide: Mano y antebrazo. Sarcoma sinovial: Proximidad de las articulaciones (rodilla o pie). Tumor fibroso solitario [12].

\subsubsection{Por la exploración física y analítica}

Dolor: La localización y el volumen de la masa tumoral influyen. Los hemangiomas, el leiomiosarcoma, y el sarcoma sinovial ocasionan dolor importante. Posterior al análisis se llega a la conclusión de que los diagnósticos más probables con hamartroma mesenquial que es un tumor benigno que se presenta en la infancia temprana y niñez. No invaden tejidos adyacentes, ni metastatizan. Se presentan como una G masa extra pleural de gran tamaño. En la tomografía computarizada se pueden identificar que es de origen óseo y la matriz (mineralizada o condroide). Tumor fibroso solitario es un tumor muy raro que tiene una incidencia menor al $5 \%$ de todos los tumores de la pleura (se han descrito menos de 800). Edad media de aparición 6-7 décadas. Solo se han descrito 2 casos en adolescentes y afecta por igual a ambos sexos. Tienen un crecimiento lento ya que son de naturaleza benigna (80\%). Suelen comprimir estructuras, menos frecuentemente las invaden. Con el tiempo pueden malignizar o metastatizar. Gran masa bien delimitada, lobulada, de atenuación/intensidad heterogénea que capta contraste en $75 \%$ de los casos. Más de $26 \%$ presenta calcificaciones. La mitad dependen de la pleura visceral y son pediculados. Sarcoma de pared toráxica que pueden originarse tanto en las partes blandas (músculos, grasa, tejido conectivo). Como de los diversos huesos de la caja torácica. Costillas, esternón, vértebras dorsales, escápulas y clavículas. La edad de presentación es variable, el sarcoma de Ewing, el tumor primitivo neuroectodérmico (tumor de Askin) y el rabdomiosarcoma se presentan en niños, adolescentes y adultos jóvenes. Los osteosarcomas y sarcomas sinoviales en adultos jóvenes ( $2^{\mathrm{a}}-3^{\mathrm{a}}$ décadas). Los condrosarcomas y fibrohistiocitomas malignos en adultos mayores ( $4^{\mathrm{a}}$ a $7^{\mathrm{a}}$ décadas). En la clínica se presentan como tumoraciones palpables dependientes de la pared torácica. Asociadas a dolor aunque, a veces, pueden ser indoloras, mal estado general, fiebre, disnea. Se presenta en paciente que han tenido antecedentes patológicos previos como, tratamientos previos como radioterapia (RT), principalmente en pacientes con antecedentes de neoplasia de mama o de pulmón [14].

El fibrohistiocitoma maligno es el sarcoma más frecuente después de RT (con menor frecuencia, el osteosarcoma también se puede originar tras RT). La existencia de alguna enfermedad de base; por ejemplo la neurofibromatosis tipo 1 o enfermedad de Von Recklinghausen se asocia a tumores malignos de la vaina nerviosa o neurofibrosarcoma. Exploración radiológica inicial en el estudio indica la presencia de una masa de pared torácica además se manifiesta una opacidad, de características extrapulmonares 
(con presencia del 'signo de la embarazada', al menos en una de las proyecciones). A veces acompañada de erosión o destrucción ósea de uno o más huesos (costillas, esternón, etc.) o bien la presencia de calcificaciones. Exploración radiológica inicial en el estudio de una masa de pared torácica. Pone de manifiesto una opacidad, de características extrapulmonares (con presencia del 'signo de la embarazada', al menos en una de las proyecciones). A veces acompañada de erosión o destrucción ósea de uno o más huesos (costillas, esternón, etc.) o bien la presencia de calcificaciones [15].

\subsection{Intervención terapéutica}

Al paciente en estudio se le realizó una biopsia de la masa toráxica cuyos hallazgos fueron: La presencia de una masa toráxica derecha de aproximadamente 10 por $18 \mathrm{~cm}$ que se extiende hacia la cavidad, de aspecto lobulado con cápsula. Presencia de derrame pleural derecho aproximadamente de $2000 \mathrm{~mL}$ de características serohemáticas. Presencia de aparentes implantes en lóbulo medio y pleura parietal. Lóbulo pulmonar colapsado por la presencia de una masa toráxica. Al paciente se le sometió a 3 sesiones de quimioterapia.

\section{Seguimiento y Resultados}

Pese al tratamiento quimioterápico ejecutado el paciente presenta metástasis. En región parietal derecha de localización extraxial se identifican lesión expansiva de bordes hiperdensos que impresiona ser dependientes de hueso el diploide se observa de aspecto permeativo de corticales irregulares, en ventana ósea múltiples imágenes osteotíticas en el diploide a nivel de la convexidad.

\section{Discusión}

El sarcoma de Ewing es un cáncer pediátrico, la edad media de aparición son los 15 años son más frecuentes en los niños, la incidencia es baja en las dos primeras décadas de vida se presentan alrededor de 3 casos por cada millón de individuos. Entre el 80 y 90 de los casos de presentan en los pacientes entre 5 y 25 años. Siendo así que el $25 \%$ de los casos se presentan en la primera década de la vida y alrededor del $65 \%$ alrededor los 10 y 20 años [16].

El pronóstico es más sombrío en los varones, los sitios frecuentes de localización son los huesos largos, se presentan con mayor frecuencia en las personas de raza blanca que en los de raza negra. Existen varias teorías que sugieren el origen de los Sarcomas de Ewing, se cree que surgen de un tejido embrionario de la cresta neural y se afirma que por características morfológicas parecidas pudieran tener origen similar al del tumor neuro ectodérmico primitivo (PNET). Pues se afirmaba que no únicamente tenían características histológicas parecidas, sino que en el $90 \%$ de los casos se presentaba la tras locación una anomalía genética $[9,17]$. 
El Sarcoma de Ewing extraóseo se presenta con mayor frecuencia en la pared toráxica. Dentro del punto de vista histológico las células neoplásicas del Sarcoma de Ewing se caracterizan por que a la tinción eosina adquieren una coloración azulada, de allí su nombre de células pequeñas redondas y azules [18, 19]. Clínicamente se manifiesta como masa dolorosa que en ocasiones se acompaña de dolor fiebre, pérdida de peso y aumento de la velocidad de sedimentación glomerular [20, 21]. El diagnóstico se realiza por hallazgos radiológicos e histológicos, en la radiografía se observa de una lesión del hueso de patrón apolillado la misma que es mal definida, a esto se suma una reacción del perioostío que se define en capas de cebolla o se pueden manifestar como rayos de un sol naciente que se acompañan de una masa que involucran partes blandas[22-24]. En la tomografía computarizada se observa una notable destrucción ósea que puede extenderse hasta la médula y a los tejidos subyacentes [25]. Mediante la resonancia magnética nuclear se puede definir afectación intra y extraósea y delimita de forma exacta la extensión [25].

Dolor: La localización y el volumen de la masa tumoral influyen. Los hemangiomas, el leiomiosarcoma, y el sarcoma sinovial ocasionan dolor importante. Posterior al análisis se llega a la conclusión de que los diagnósticos más probables con hamartroma mesenquial que es un tumor benigno que se presenta en la infancia temprana y niñez. No invaden tejidos adyacentes, ni metastatizan. Se presentan como una $\mathrm{G}$ masa extra pleural de gran tamaño. En la tomografía computarizada se pueden identificar que es de origen óseo y la matriz (mineralizada o condroide). Tumor fibroso solitario es un tumor muy raro que tiene una incidencia menor al $5 \%$ de todos los tumores de la pleura (se han descrito menos de 800). Edad media de aparición 6-7 décadas. Solo se han descrito 2 casos en adolescentes y afecta por igual a ambos sexos. Tienen un crecimiento lento ya que son de naturaleza benigna (80\%). Suelen comprimir estructuras, menos frecuentemente las invaden. Con el tiempo pueden malignizar o metastatizar. Gran masa bien delimitada, lobulada, de atenuación/intensidad heterogénea que capta contraste en $75 \%$ de los casos. Más de $26 \%$ presenta calcificaciones. La mitad dependen de la pleura visceral y son pediculados. Sarcoma de pared toráxica que puede originarse tanto en las partes blandas (músculos, grasa, tejido conectivo). Como de los diversos huesos de la caja torácica. Costillas, esternón, vértebras dorsales, escápulas y clavículas. La edad de presentación del sarcoma de Ewing es variable. El tumor primitivo neuroectodérmico (tumor de Askin) y el rabdomiosarcoma se presentan en niños, adolescentes y adultos jóvenes. Los osteosarcomas y sarcomas sinoviales en adultos jóvenes ( $2^{\mathrm{a}}-3^{\mathrm{a}}$ décadas). Los condrosarcomas y fibrohistiocitomas malignos en adultos mayores ( $4^{\mathrm{a}}$ a $7^{\mathrm{a}}$ décadas). En la clínica se presentan como tumoraciones palpables dependientes de la pared torácica. Asociadas a dolor aunque, a veces, pueden ser indoloras, producen mal estado general, fiebre, disnea. Se presenta en pacientes que han tenido antecedentes patológicos previos, como tratamientos de radioterapia (RT), principalmente en aquellos con antecedentes de neoplasia de mama o de pulmón [14].

El fibrohistiocitoma maligno es el sarcoma más frecuente después de RT (con menor frecuencia, el osteosarcoma también se puede originar tras RT). La existencia de alguna enfermedad de base; por ejemplo la neurofibromatosis tipo 1 o enfermedad de Von 
Recklinghausen que se asocia a tumores malignos de la vaina nerviosa o neurofibrosarcoma. La exploración radiológica inicial del estudio indica la presencia de una masa de pared torácica, además se manifiesta una opacidad, de características extrapulmonares (con presencia del 'signo de la embarazada', al menos en una de las proyecciones). A veces acompañada de erosión o destrucción ósea de uno o más huesos (costillas, esternón, etc.) o bien la presencia de calcificaciones. Exploración radiológica inicial en el estudio de una masa de pared torácica. Pone de manifiesto una opacidad, de características extrapulmonares (con presencia del 'signo de la embarazada', al menos en una de las proyecciones). A veces acompañada de erosión o destrucción ósea de uno o más huesos (costillas, esternón, etc.) o bien la presencia de calcificaciones [15].

\subsection{Perspectiva del paciente}

La supervivencia del paciente por las metástasis que presenta se considera que es de alrededor de 5 años.

\section{Agradecimientos}

Al paciente y a su familia permitirnos dar a conocer su historia de vida y ser un ejemplo de fortaleza ante la adversidad. Los investigadores informan no poseer conflicto de intereses.

\section{Consentimiento Informado}

Se obtuvo el consentimiento informado a través del representante legal del paciente pues es menor de edad.

\section{References}

[1] Resnick D, Kyriakos M. Tumors and tumor-like lesions of bone: Imaging and pathology of specific lesions. Philadelphia: Saunders; 2002. 4060-4065 p.

[2] Fuentes E, Tornes K, Fuentes J. Sarcoma de ewing nasosinusal en la infancia. Multimed rRevista Médica Granma. 2020;24(1):190-9.

[3] Baruchel S, Pappo A, Krailo M, et al. A phase 2 trial of trabectedin in children with recurrent rhabdomyosarcoma, Ewing sarcoma and non-rhabdomyosarcoma soft tissue sarcomas: A report from the Children's Oncology Group. Eur J Cancer. 2012;48(4):579-85.

[4] Collier AB, Simpson L, Monteleone P. Cutaneous Ewing sarcoma: Report of 2 cases and literature review of presentation, treatment, and outcome of 76 other reported cases. J Pediatr Hematol Oncol. 2011;33(8):631-4.

[5] Antelo JS, García CR, Martínez CM, Hernando HV. Sarcoma de Ewing pulmonar/tumor neuroectodérmico primitivo (PNET): aportación de un caso y revisión de la bibliografía. Arch Bronconeumol. 2010;46(1):44-6.

[6] Tirode F. Biology of Ewing sarcoma. Bone cancer prim bone cancers bone metastases. $2^{\text {nd }}$ ed. 2015;2013:245-55.

[7] Grünewald TGP, Cidre-Aranaz F, Surdez D, et al. Ewing sarcoma. Nat Rev Dis Prim. 2018;4(1).

[8] Crompton BD, Stewart C, Taylor-Weiner A, et al. The genomic landscape of pediatric Ewing sarcoma. Cancer Discov. 2014;4(11):1326-41.

[9] Ladenstein R, Pötschger U, Le Deley MC, et al. Primary disseminated multifocal Ewing sarcoma: Results of the Euro-EWING 99 trial. J Clin Oncol. 2010;28(20):3284-91. 
[10] Jiménez E, Chinchilla L, Gómez A, Avilés A, Martínez A. Sarcoma de mediastino. Rev la Fac la UNAM. 2017;60(3):6-17.

[11] Dini LI, Mendonça R, Gallo P. Primary Ewing's sarcoma of the spine: Case report. Arq Neuropsiquiatr. 2006;64(3 A):654-9.

[12] Machado I, Cruz J, Lavernia J, et al. Superficial EWSR1-negative undifferentiated small round cell sarcoma with CIC/DUX4 gene fusion: A new variant of Ewing-like tumors with locoregional lymph node metastasis. Virchows Arch. 2013;463(6):837-42.

[13] Bendahan V, Tejada J, Gómez C, et al. Localizaciones atípicas del Sarcoma de Ewing: A propósito de dos casos Objetivo docente. Seram. 2012;1-17.

[14] Millán E, Corona R, Ríos N. Sarcoma de Ewing en paciente masculino Ewing's sarcoma in a male patient. Rev Cuba Med Gen Integr. 2010;26(3):569-75.

[15] Vivas-Flores Cl, Pantoja-Garrido M, Frías-Sánchez Z. Extraosseous Ewing's sarcoma of gynaecological origin: Primitive neuroectodermal tumour of infrequent location. Case report and review of the literature. Rev Colomb Obstet Ginecol. 2017;68(2):135-41.

[16] Esiashvili N, Goodman M, Marcus RB. changes in incidence and survival of ewing sarcoma patients over the past 3 decades. J Pediatr Hematol Oncol. 2008;30(6):425-30.

[17] Hauben E, Van de Broek L, Van Marck E, Hogendoorn P. Adamantinoma-like Ewing's sarcoma and Ewing's-like. J Pathol. 2001;195:218-21.

[18] Hisaoka M, Matsuyama A, Nagao $\mathrm{Y}$, et al. Identification of Altered MicroRNA Expression Patterns in Synovial Sarcoma. Cancer. 2011;396(January):389-96.

[19] Catalan J, Fonte AC da, Lusa JRB, Oliveira AD de, Melo ES de, Gonçalves CM. Sarcoma de Ewing: Aspectos clínicos e radiográficos de 226 casos. Radiol Bras. 2005;38(5):333-6.

[20] Cotterill SJ, Ahrens S, Paulussen M, et al. Prognostic factors in Ewing's tumor of bone: Analysis of 975 patients from the European Intergroup Cooperative Ewing's Sarcoma Study Group. J Clin Oncol. 2000;18(17):3108-14.

[21] Murphey MD, Senchak LT, Mambalam PK, Logie Cl, Klassen-Fischer MK, Kransdorf MJ. From the radiologic pathology archives: Ewing sarcoma family of tumors: Radiologic-pathologic correlation. Radiographics. 2013;33(3):803-31.

[22] Batsakis JG, El-Naggar AK. Ewing's sarcoma and primitive neuroectodermal tumors. Adv Anat Pathol. 1997;4(4):207-20.

[23] Souvirón Encabo R, Arenas Britez O, Gil R, Pérez-Luengo E, Scola Yurrita B. Tumor neuroectodérmico primitivo periférico/Sarcoma de Ewing Extraóseo del hueso temporal. Acta Otorrinolaringol Esp. 2005;56(1):38- 40.

[24] Riggi N, Stamenkovic I. The Biology of Ewing sarcoma. Cancer Lett. 2007;254(1):1-10.

[25] Kumagai A, Motoi T, Tsuji K, Imamura T, Fukusato T. Detection of SYT and EWS gene rearrangements by dual- color break-apart $\mathrm{CISH}$ in liquid-based cytology samples of synovial sarcoma and Ewing sarcoma/primitive neuroectodermal tumor. Am J Clin Pathol. 2010;134(2):323-31. 